\title{
Mid-IR laser for wavefront correction in gravitational wave detectors
}

Beniwal, Deeksha, Cao, Huy, Ng, Sebastian, Brooks, Aidan, Bharathan, Gayathri, et al.

Deeksha Beniwal, Huy T. Cao, Sebastian Ng, Aidan F. Brooks, Gayathri Bharathan, Alex Fuerbach, Peter J. Veitch, David J. Ottaway, "Mid-IR laser for wavefront correction in gravitational wave detectors," Proc. SPIE 11200, AOS Australian Conference on Optical Fibre Technology (ACOFT) and Australian Conference on Optics, Lasers, and Spectroscopy (ACOLS) 2019, 112002C (30 December 2019); doi: 10.1117/12.2540061 


\title{
Mid-IR laser for wavefront correction in gravitational wave detectors
}

\author{
Deeksha Beniwal*a, Huy T. Cao ${ }^{\mathrm{a}}$, Sebastian Nga , Aidan F. Brooks ${ }^{\mathrm{b}}$, Gayathri Bharathan ${ }^{\mathrm{c}}$, Alex \\ Fuerbach $^{\mathrm{c}}$, Peter J. Veitch and David J. Ottaway ${ }^{\mathrm{a}}$ \\ ${ }^{a}$ University of Adelaide, SA 5001, Australia; ${ }^{b}$ California Institute of Technology, Pasadena, CA, \\ 91125; 'Macquarie University, NSW 2109, Australia
}

The next-generation gravitational wave detectors aim to enhance our understanding of extreme phenomena in the Universe. The high-frequency sensitivity of these detectors will be maximized by injecting squeezed vacuum states into the detector. However, the performance advantages offered by squeezed state injection can be easily degraded by losses in the system ${ }^{1,2}$. A significant source of loss is the mode mismatch between optical cavities within the interferometer. To overcome this issue, new actuators are required that can produce a highly spherical wavefront change, with minimal higher order aberrations, whist adding low phase noise to the incident beam.

We propose exploiting the residual $\mathrm{OH}$ in the fused silica mirrors, which have a strong absorption feature in the 2.7$2.8 \mu \mathrm{m}$ range, to allow active correction of this mode mismatch. This work is the first demonstration of a spherical wavefront correction using a mid-infrared fibre laser for actuation in place of the more routinely used $\mathrm{CO}_{2}$ laser. It also paves the way for the use of longer wavelength fibre lasers that rely on the intrinsic absorption of fused-silica and hence could be used for ultra-low $\mathrm{OH}$ fused silica.

We developed a wavelength stabilised, watt-level, Er:ZBLAN fibre laser system to target the $\mathrm{OH}$ absorption in UV fused silica mirrors. The peak emission wavelength of the laser was chosen to be $2785 \mathrm{~nm}$ to avoid absorption by water vapour. In the laser setup shown in Figure 1 (left), a 1.6m piece of Er-doped ZBLAN fibre is pumped using a 980nm diode in a double pass configuration. The laser cavity is formed by two fibre Bragg gratings (FBGs) with reflectivities of $98.4 \%$ and $10 \%$. These were written into the core of the fibre using an ultra-low loss, direct femtosecond laser inscription method developed at Macquarie University ${ }^{3}$. The laser system has a slope efficiency of $14 \%$ and a peak emission wavelength of $2785.2 \pm 0.1 \mathrm{~nm}$ (See Figure 1 Right) with a pump-power tuning coefficient of $0.04 \mathrm{~nm} / \mathrm{W}$, which results in negligible changes in the absorption in fused silica. Long term fluctuations in the output laser power are approximately $2 \%$ at a range of output power levels without pump stabilisation.
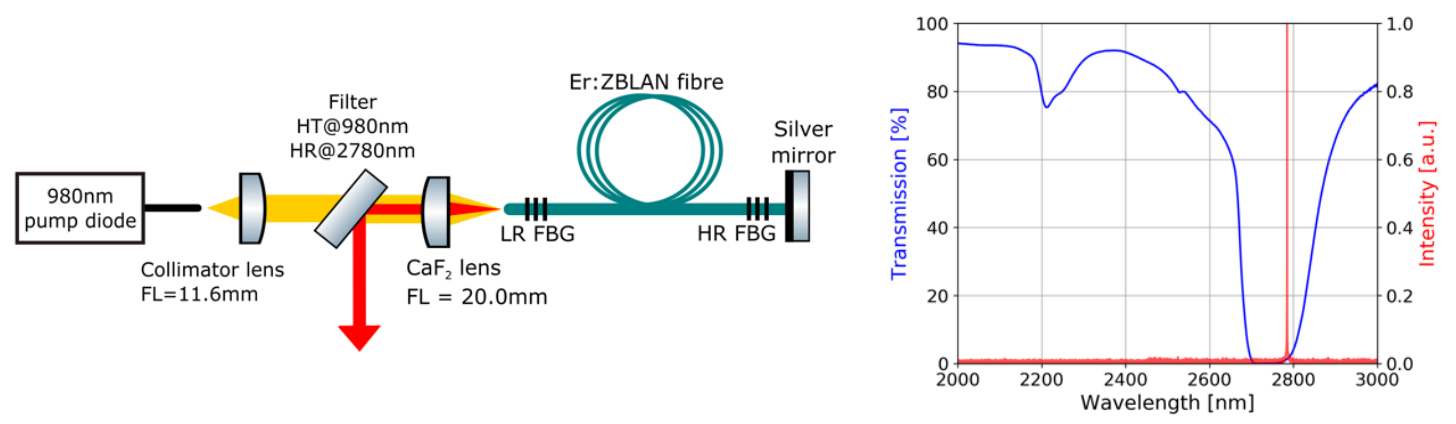

Figure 1. Left: The setup of the diode-pumped $2.78 \mu \mathrm{m}$ fibre laser. The yellow beam shows the path taken by the pump light while the red beam shows the path of laser light. Right: Transmission spectrum of a 10mm thick, UV fused silica optic (blue) with the laser emission spectrum (red) overlaid.

The measurement system used to test the thermal actuation technique is shown in Figure 2. The output of the Er:ZBLAN fibre laser is incident on a UV fused silica optic suspended in a vacuum. The thermal lens induced in the optic is measured using a differential Hartmann wavefront sensor (HWS) ${ }^{4}$. The HWS consists of an array of holes in an opaque plate that is mounted on a CCD camera. An incident probe beam - from the 680nm SLED - produces an array of spots on the camera. Changes in the positions of the spots are used to determine the change in the incident wavefront.

*deeksha.beniwal@adelaide.edu.au;

phone $+6188313-7732$;

AOS Australian Conference on Optical Fibre Technology (ACOFT) and Australian Conference on Optics, Lasers, and Spectroscopy (ACOLS) 2019, edited by Arnan Mitchell, Halina Rubinsztein-Dunlop, Proc. of SPIE Vol. 11200, 112002C · C 2019 SPIE · CCC code: 0277-786X/19/\$21 - doi: 10.1117/12.2540061 


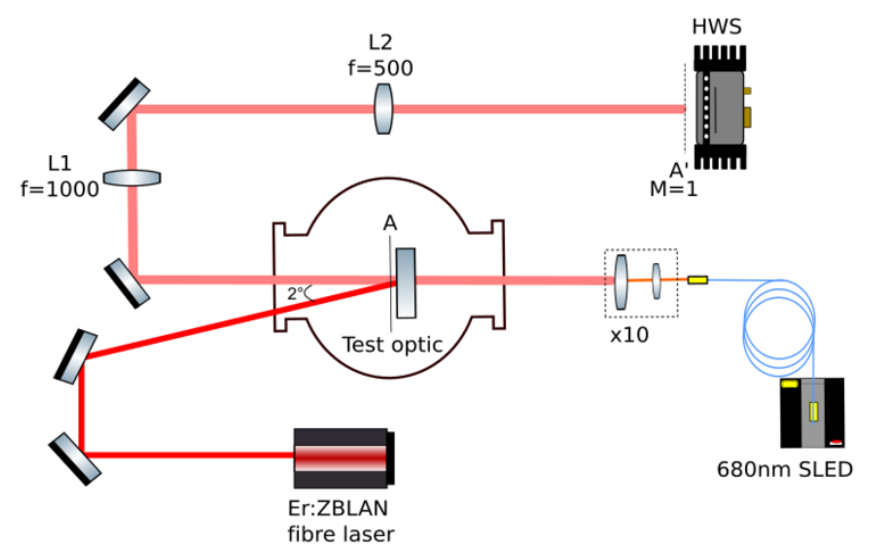

Figure 2. Schematic of the setup used to measure the wavefront changes induced by absorption of laser beam from Er:ZBLAN laser. The SLED output is used to illuminate the heated region of the test optic. The rear surface of the optic is imaged onto the Hartmann Wavefront Sensor.

The results of a preliminary experiment are shown in Figure 3. The noise floor of the HWS is due to photoelectron shot noise, resulting in wavefront-change sensitivity of $<5$ nmRMs. The observed wavefront distortion was highly quadratic: an absorbed power of $0.35 \mathrm{~W}$ produced $27 \pm 1 \mathrm{mD}$ of spherical power and $3 \pm 1 \mathrm{mD}$ of cylindrical power. The spherical power increased linearly with incident laser power. Subtraction of quadratic components revealed low levels of higher-order aberrations.
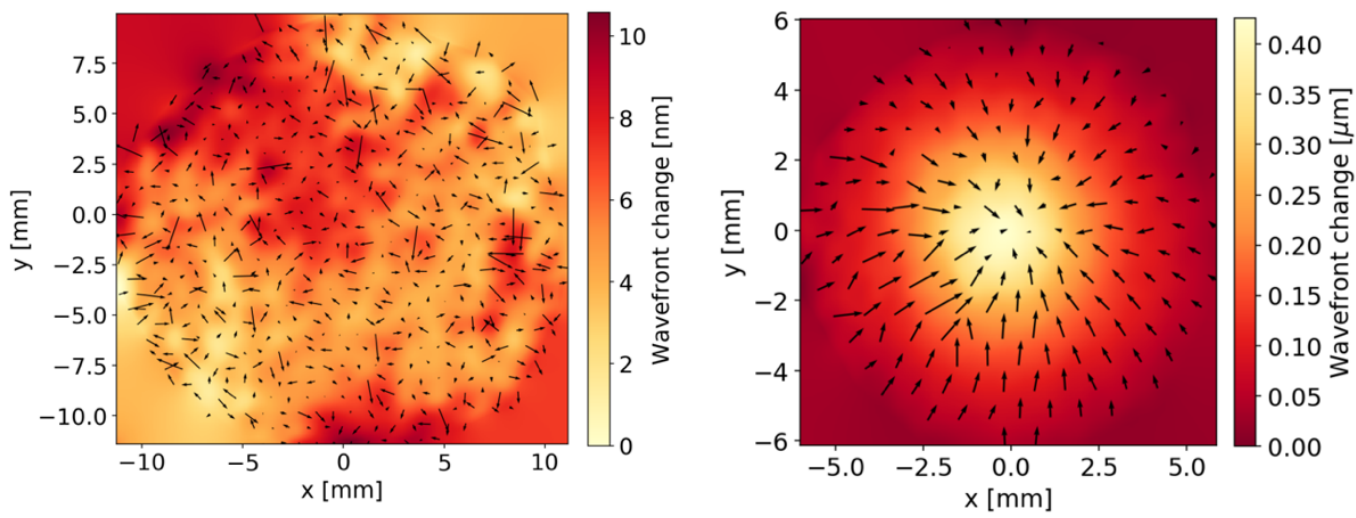

Figure 3. Left. The measured gradient field (black arrows) and the reconstructed wavefront noise floor of the HWS. Right.

Gradient field and wavefront change due to $0.35 \mathrm{~W}$ of incident laser power at $2.78 \mu \mathrm{m}$.

In conclusion, we developed a watt-level Er:ZBLAN fibre laser with emission near the centre of the 2.7-2.8 $\mu \mathrm{m}$ OH absorption band in UV fused silica. We demonstrated that the output could be used to produce a thermal lens in a transmissive fused silica optic with only low levels of higher order aberrations and with no mechanical or electrical contact, as required for active mode matching in advanced gravitational wave detectors.

\section{REFERENCES}

[1] Mansell G., "Squeezed light sources for current and future gravitational-wave detectors," GWADW, LIGOG1602336-v2 (2017)

[2] Oelker E., L. Barsotti, S. Dwyer, D. Sigg, N. Mavalvala, "Squeezed light for advanced gravitational wave detectors and beyond," Opt. Express 22, (2014)

[3] Bharathan G., T.T. Fernandez, M. Ams, R. I. Woodward, D. D. Hudson, and A. Fuerbach, "Optimized laser-written ZBLAN fiber Bragg gratings with high reflectivity and low loss," Opt. Lett. 44, 423-426 (2019)

[4] Brooks A. F., et al., "Ultra-sensitive wavefront measurement using a Hartmann sensor," Opt. Express 15, 10370 (2007) 